\title{
Subarachnoid hematoma after spinal anesthesia - A case report -
}

Received August 29, 2017

Revised October 18, 2017

Accepted October 19, 2017

\section{Corresponding author}

Yong-Hyun Cho, M.D.

Department of Anesthesiology and

Pain Medicine, Seoul Sungsim

General Hospital, 259 Wangsan-

ro, Dongdaemun-gu, Seoul 02488,

Korea

Tel: 82-2-966-1616

Fax: 82-2-968-2394

E-mail: anesthecho@naver.com

ORCID

http://orcid.org/0000-0002-8323-1933

\section{Won Jang, Yong-Hyun Cho, Dong-Hyun Lee, and Sun-Hee Kim}

Department of Anesthesiology and Pain Medicine, Seoul Sungsim General Hospital, Seoul, Korea

Spinal subarachnoid hematoma is a very rare complication of spinal anesthesia. This complication can, and is, often overlooked and dismissed as a fatal neurological consequence of (what amounts to) delayed diagnosis. In this case, a 59-year-old female patient with no specific medical history underwent right knee arthroscopy under spinal anesthesia. The arthroscopic surgery concluded without complications but, on the first postoperative day, the patient complained of lower back pain, headache, nausea, vomiting. On the fifth postoperative day, magnetic resonance imaging was taken and it revealed evidence of a subarachnoid hematoma involving the L3 and L4 vertebral levels. Hematoma evacuation was performed, and the patient recovered without sequelae. Here, we report this case that lumbar spinal subarachnoid hematoma was found five days after spinal anesthesia which was done in a patient without coagulopathy.

Key Words: Spinal anesthesia, Spinal subarachnoid hematoma.
Generally, the incidence of spinal hematoma is rare, but the condition does occur and due to acute and gradual progression, neurological damage or even death can occur if the problem is not diagnosed timely and then treated appropriately. The incidence of spinal hematoma after spinal anesthesia is maximally $1: 220,000$, and it can be classified as most common epidural (75\%), subarachnoid (15.7\%) and subdural (4.1\%) depending on the location [1]. Jeon et al. [2] explained that subarachnoid hematoma is rare because bleeding is diluted by cerebrospinal fluid and washed away by flow. Underlying medical conditions are often encountered in patients affected by this complication. These incidentally diagnosed pathologies include hematological malignancies, bleeding disorders or therapeutic anticoagulation [3]. However, spinal hematoma is very rare in patients without coagulopathy [4]. Herein, we will to analyze and discuss a case of a subarachnoid hematoma which developed after spinal anesthesia in a patient without any significant medical history, and also emphasize the importance of early diagnosis for minimizing neurological damage.

\section{CASE REPORT}

This 59-year-old female patient with no specific medical history underwent right knee arthroscopy under spinal anesthesia. The patient was $158 \mathrm{~cm}$ tall and weighed $64 \mathrm{~kg}$. Preoperatively, platelet counts were $326,000 / \mathrm{mm}^{3}$, prothrombin time was 10.1 seconds, and activated partial thromboplastin time was 25.9 seconds. These were all within normal range. Spinal anesthesia was successfully initiated on the first attempt, and accomplished without difficulty. A 23-gauge Quincke needle was inserted at L3-4. Clear cerebrospinal fluid (CSF) was observed and $13 \mathrm{mg}$ of $0.5 \%$ hyperbaric bupivacaine was injected. No paresthesia was reported by the patient during the entire procedure, and no blood was obtained.

The patient switched back to the supine position. After one minute, the sensory block was T10, and about fifteen minutes

This is an Open Access article distributed under the terms of the Creative Commons Attribution Non-Commercial License (http://creativecommons.org/licenses/by-nc/4.0) which permits unrestricted non-commercial use, distribution, and reproduction in any medium, provided the original work is properly cited.

Copyright (c) the Korean Society of Anesthesiologists, 2018 
after taking the head-up position, the sensory block was T9. The operation proceeded without undue complication, and the patient was moved to the recovery ward. The duration of anesthesia was four hours, and the patient had no neurological symptoms and safely recovered from anesthesia.

On the first postoperative day, the patient complained of lower back pain, headache, nausea, and vomiting, but motor and sensory function were intact. Postural adjustments had no effect on the patient's headache, and the pain was continual and unrelenting. Conservative treatments including acetaminophen and tramadol were administered in an effort to treat the transient neurologic symptoms. The patient's symptoms did not respond to conservative therapy and remained unchanged until four days postoperatively. Body temperature was maintained at $37.1^{\circ} \mathrm{C}$ and $37.3^{\circ} \mathrm{C}$ and C-reactive protein (CRP) level was $0.1 \mathrm{mg} / \mathrm{dl}$.

On the fifth postoperative day, the patient complained of neck stiffness, there was a positive Kernig sign and her body temperature was $38.7^{\circ} \mathrm{C}$. As well, the CRP level had increased to $2.4 \mathrm{mg} / \mathrm{dl}$. Magnetic resonance imaging (MRI) was taken in an effort to differentiate between epidural abscess, meningitis and epidural hematoma. The MRI ultimately revealed evidence of a subarachnoid hematoma (Figs. 1A and 1B). The hematoma was located ventral to the spinal cord and encompassed the entire L3 to L4 vertebral level. The size of the hematoma was very large $(1.5 \mathrm{~cm} \times 12 \mathrm{~cm})$. Therefore, the decision was reached to evacuate the hematoma before the additional neurologic symptoms appeared.

With the patient in prone position, a midline incision was made over the spinous process of L2-L4. The spinous processes of L3 and L4 were debrided and decompressive laminectomies were performed, bilaterally. The dura mater was exposed and its color was noted to be dark red. A needle puncture site was not found on the dura mater surface, however. The CSF was aspirated with $23 \mathrm{G}$ needle and reddish fluid was aspirated (Fig. 2). The cauda equina was bifurcated at midline and retracted to both sides. Under the spinal nerve rootlets, there was a clotted mass of blood (the hematoma) (Fig. 3). All of hematoma was removed. During the operation, a blood culture was obtained. No evidence of infection was found in the results after three weeks.

After surgery, the patient reported decreased nausea, vomiting, neck stiffness and lower back pain, but the headache showed a persistent pattern and additional conservative management was performed. On the 14th postoperative day, another MRI was taken for follow-up. All evidence of the hematoma had disappeared and all the remaining symptoms

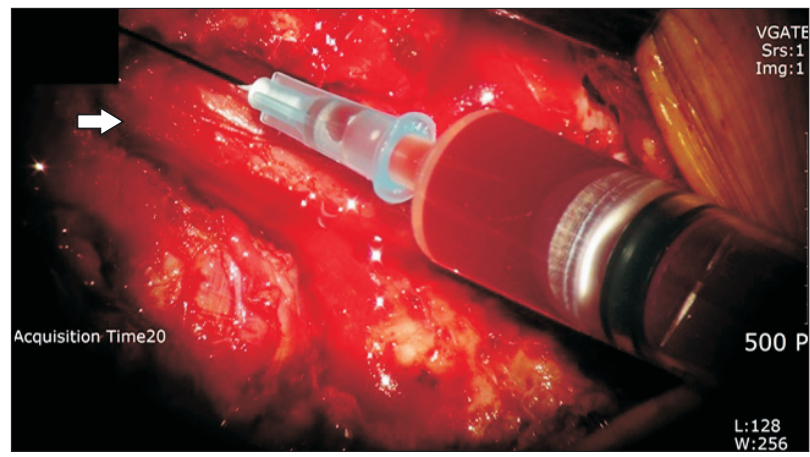

Fig. 2. Intraoperative photograph showed that dura mater (arrow) was exposed and its color was dark red. Cerebrospinal fluid was aspirated with $23 \mathrm{G}$ needle and reddish fluid was aspirated.
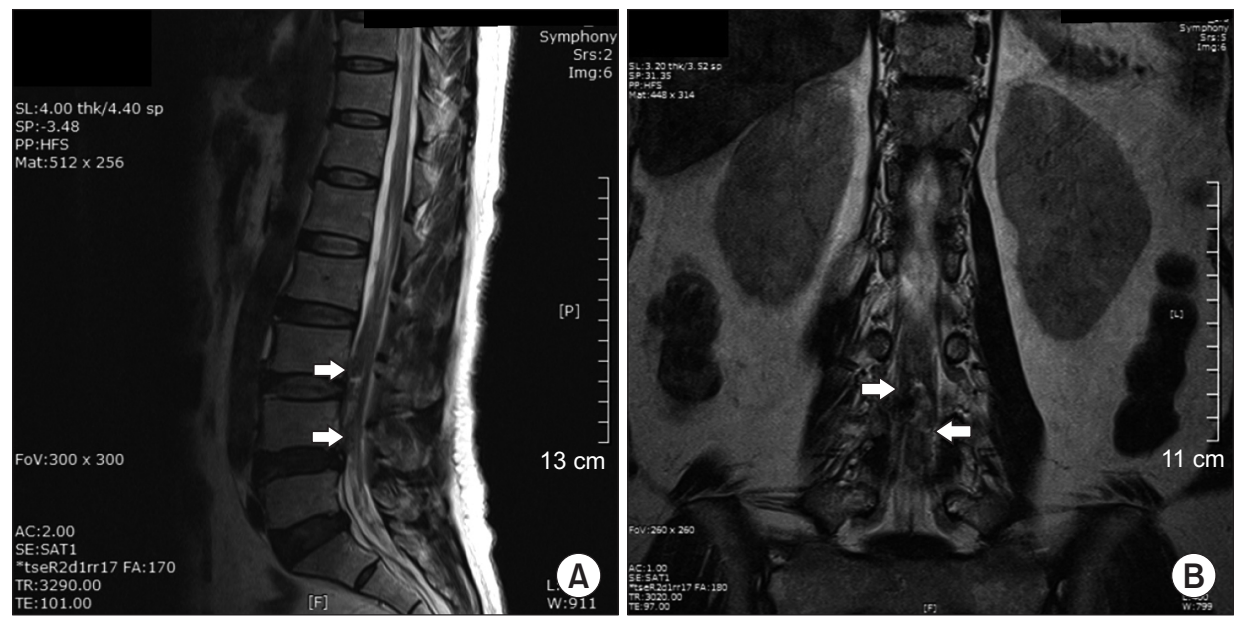

Fig. 1. (A, B) Lumbar magnetic resonance imaging ( $T 2$ weighted image) showed heterogeneous signal intensity (arrows) at ventral to the spinal cord spreading from L3 to L4. 
had subsided (Figs. 4A and 4B).

\section{DISCUSSION}

Subarachnoid hematoma is less common than epidural hematoma. However, prompt diagnosis of the condition is important because the hematoma can directly compress the spinal cord or cauda equine and produce serious secondary neurological disorders. Domenicucci et al. [5] reviewed 69 cases of spinal subarachnoid hematoma. They concluded that spinal subarachnoid hematoma is rare after lumbar puncture, but that lumbar puncture is a major cause in patients with coagulopathies due to pharmacologic treatment and systemic disease. In addition, subarachnoid hematoma was reported to be the most prevalent in technically difficult issues encountered with spinal anesthesia. The cause of hemorrhage is usually the perforation of the radicular vessels

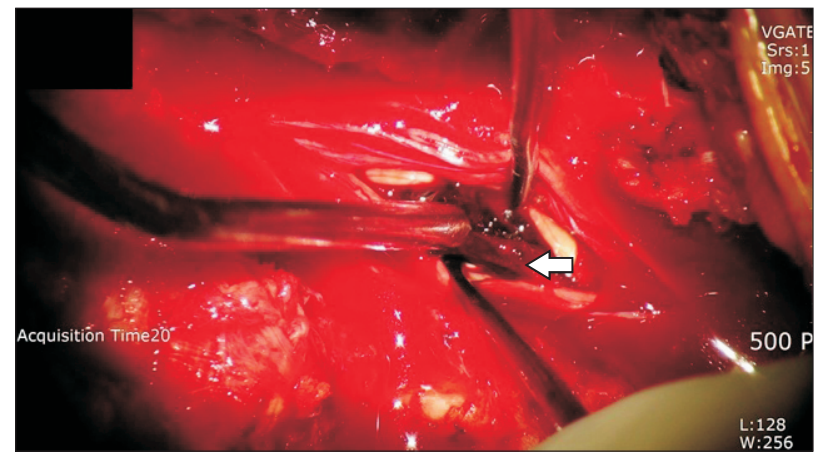

Fig. 3. Intraoperative photograph showed that under the rootlets, there was clotted mass of hematoma (arrow). in the arachnoid space, and it has been speculated that the passage of the blood into the subdural space usually occurs via lacerations in the arachnoid due to the intradural punctures [6].

Because the patient did not have any reported risk factors which would render her susceptible to development of subarachnoid hematoma, the authors questioned whether there was a causative factor associated with the technique. Because the hematoma was confined to the ventral portion of the subarachnoid space, we suspected that the needle penetrated deeper into the anterior portion of the subarachnoid space during spinal anesthesia. In addition, needling that was not gentle could certainly increase the possibility of bleeding.

After developing back pain of acute onset, one-third of patients with spinal subarachnoid hematoma also exhibit meningeal irritation signs such as headache, vomiting, neck stiffness and opisthotonus [1]. The headache in this patient was not considered to be post-dural puncture headache because there was no change with posture. Minor symptoms of small subarachnoid hematomas can be misdiagnosed as transient neurologic symptom (TNS). The significant difference between the two for differential diagnosis is the duration of the symptoms. Most symptoms of TNS disappear within two days [6]. In this case, however, the symptom of spinal hematoma was continually present until the patient was five days postoperative. As well, the patient also had a fever of $38.7^{\circ} \mathrm{C}$ and meningeal irritation signs at five days postoperatively. The meningeal irritation sign is likely to be caused by a secondary local inflammatory reaction to blood or hemoglobin degradation products in the intrathecal space [7]. The value
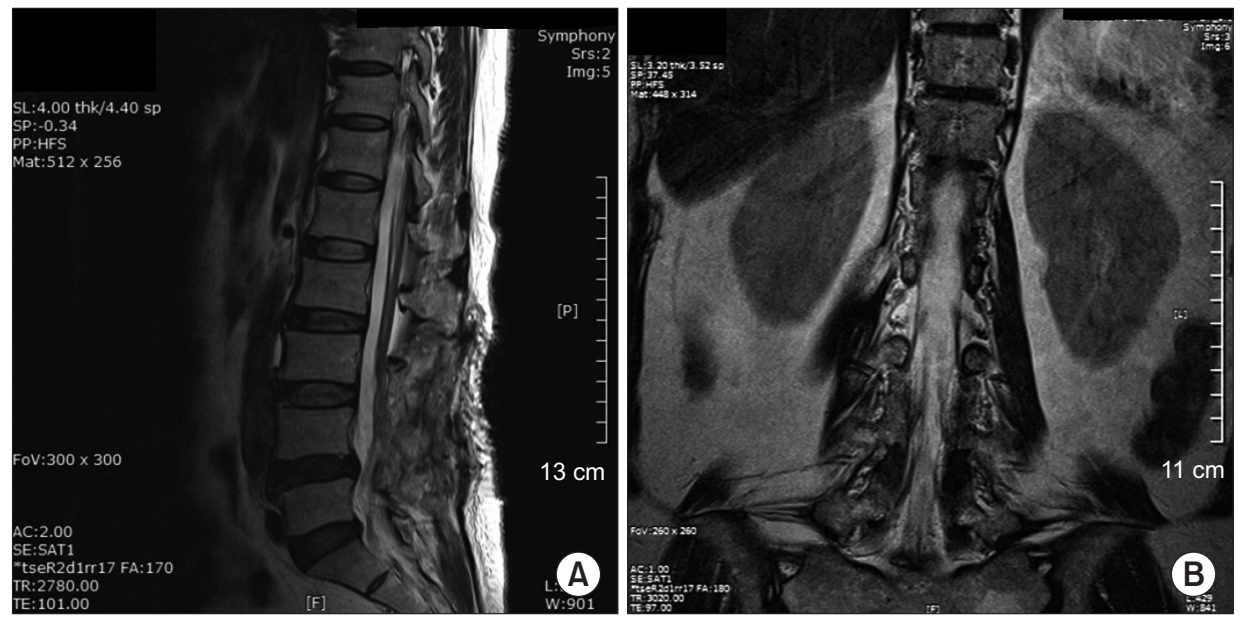

Fig. 4. (A, B) Postoperative lumbar magnetic resonance imaging showed complete evacuation of the subarachnoid hematoma. 
of CRP $2.4 \mathrm{mg} / \mathrm{dl}$ is probably a reasonable number after the knee arthroscopy, but the reason for the late increase is probably due to the local inflammation described above [8].

Spinal MRI is a sensitive diagnostic tool, and epidural hematoma is a major differential diagnosis of subarachnoid hematoma, especially when the degenerative changes are so severe that it distorts the anatomy of the intra-spinal compartments [9]. Although myelography is a good test for diagnosic purposes, MRI scanning was chosen in this case because myelography can, in and of itself, produce a spinal hematoma [10].

Patients with subarachnoid hematoma and severe neurological symptoms, such as paresis or sphincter dysfunction, should undergo surgical decompression [11]. The outcome of subarachnoid hematoma depends on neurological status before hematoma is released $[4,10]$. Although this patient had no neurological symptom, the surgical approach of the recently published case was performed after the appearance of severe neurological symptom such as "saddle" hypoesthesia, urinary incontinence, and postoperative prognosis was poor [11].

Therefore, hematomas have been shown to occur in patients without any remarkable contributory history of use of coagulants, multiple or traumatic centesis trial as a complication of spinal or epidural anesthesia. To avoid damage to the surrounding structures during spinal anesthesia, the CSF leak should be checked and the needle should be slowly advanced. And reasonable suspicion, early diagnosis, and quick decision of surgery should be very important for the prognosis of the patient.

\section{REFERENCES}

1. Kreppel D, Antoniadis G, Seeling W. Spinal hematoma: a litera- ture survey with meta-analysis of 613 patients. Neurosurg Rev 2003; 26: 1-49.

2. Jeon SB, Ham TI, Kang MS, Shim HY, Park SL. Spinal subarachnoid hematoma after spinal anesthesia. Korean J Anesthesiol 2013; 64: 388-9.

3. Koyama S, Tomimatsu T, Kanagawa T, Sawada K, Tsutsui T, Kimura T, et al. Spinal subarachnoid hematoma following spinal anesthesia in a patient with HELLP syndrome. Int J Obstet Anesth 2010; 19: 87-91.

4. Domenicucci M, Ramieri A, Ciappetta P, Delfini R. Nontraumatic acute spinal subdural hematoma: report of five cases and review of the literature. J Neurosurg 1999; 91(1 Suppl): 65-73.

5. Domenicucci M, Ramieri A, Paolini S, Russo N, Occhiogrosso G, Di Biasi C, et al. Spinal subarachnoid hematomas: our experience and literature review. Acta Neurochir (Wien) 2005; 147: 741-50.

6. Lam DH. Subarachnoid haematoma after spinal anaesthesia mimicking transient radicular irritation: a case report and review. Anaesthesia 2008; 63: 423-7.

7. Tjandra JJ, Varma TR, Weeks RD. Spinal arachnoiditis following subarachnoid haemorrhage. Aust N Z J Surg 1989; 59: 84-7.

8. Bruce-Brand RA, Colleran GC, Broderick JM, Lui DF, Smith EM, Kavanagh EC, et al. Acute nontraumatic spinal intradural hematoma in a patient on warfarin. J Emerg Med 2013; 45: 695-7.

9. Wang C, Ao Y, Fan X, Wang J, Cui G, Hu Y, et al. C-reactive protein and erythrocyte sedimentation rate changes after arthroscopic anterior cruciate ligament reconstruction: guideline to diagnose and monitor postoperative infection. Arthroscopy 2014; 30: 1110-5.

10. Sather MD, Gibson MD, Treves JS. Spinal subarachnoid hematoma resulting from lumbar myelography. AJNR Am J Neuroradiol 2007; 28: 220-1.

11. Avecillas-Chasín JM, Matias-Guiu JA, Gomez G, Saceda-Gutierrez J. A case of acute spinal intradural hematoma due to spinal anesthesia. J Aucte Dis 2015; 4: 341-3. 